\title{
Stability and Safety Estimates and Tests of a Superconducting Bus-Line for Large-Scale Superconducting Coils
}

\author{
Toshiyuki Mito, Shuichi Yamada, Hirotaka Chikaraishi, Kazuya Takahata, Nagato Yanagi, Akifumi Iwamoto, \\ Arata Nishimura, Shugo Tanahashi, Osamu Motojima, and Junya Yamamoto \\ National Institute for Fusion Science (NIFS), 322-6 Oroshi, Toki, Gifu 509-52, Japan
}

Toshio Uede, Hisaaki Hiue, Kazuo Ueda, and Ikuo Itoh Fuji Electric Co. Ltd., Kawasaki 210, Japan

\begin{abstract}
We have been developing a flexible superconducting bus-line as a unit electrical feeder between large-scale superconducting coils and their power supplies away from the coils. The designed superconducting bus-line consists of a pair of $+/$ aluminum stabilized NbTi/Cu compacted strand cables and a coaxial four-channel transfer line. A fullscale model of the $\mathrm{SC}$ bus-line $(20 \mathrm{~m}$ long) has been constructed and tested successfully up to $40 \mathrm{kA}$ without a quench under the short-circuit condition. Stability tests were also done by inducing a forced quench with heaters. A minimum propagation current larger than $32.5 \mathrm{kA}$ was confirmed. Thus, the bus-line was cryogenically stabilized at the rated current of $30 \mathrm{kA}$. We have examined the test results and evaluated the stability and safety margins of this bus-line. The design criteria for a superconducting bus-line are also shown for largescale superconducting coils with operating current as a parameter.
\end{abstract}

\section{INTRODUCTION}

In scaling up superconducting coils, it becomes difficult to place and install large power supplies and current-leads near the coils because of spatial restrictions. For the case of the Large Helical Device (LHD) [1], which is a heliotron/torsatron type fusion experimental device under construction at the National Institute for Fusion Science (NIFS), the distances between the superconducting coils and the power supplies are $45-65 \mathrm{~m}$. The superconducting coils for LHD consist of two helical coils (each coil has 3 blocks) and three pairs of poloidal coils (IV, IS and OV coils). We have been developing a flexible superconducting bus-line for LHD to connect the coils and the power supplies without energy consumption. The total number of superconducting buslines for LHD is nine, of which six are for the helical coils and three are for the poloidal coils. The operating current of the helical coils is $17.3 \mathrm{kA}$. The operating currents of poloidal coils IV, IS and OV are $20.3 \mathrm{kA}, 21.3 \mathrm{kA}$ and 31.3 kA, respectively.

The superconducting bus-line requires excellent stability and safety exceeding that of the superconducting coils themselves because the large magnetic stored energy of the coils must be extracted through the bus-line if and when the coils quench.

\section{DESIGN AND CONSTRUCTION OF A SUPERCONDUCTING BUS-LINE}

Manuscript received October 18, 1994.

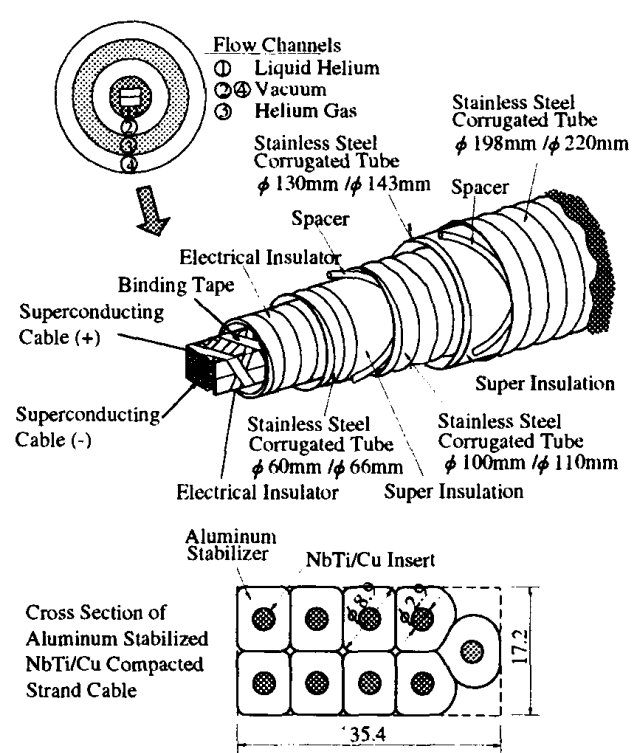

Fig. 1. Configuration of a flexible superconducting bus-line.

A full-scale model with a length of $20 \mathrm{~m}$ was constructed to investigate the feasibility and performance of the flexible superconducting bus-line [2]. The configuration and the specifications of the bus-line are shown in Fig. 1 and Table 1 , respectively. A +/- pair of superconducting cables is separated with Nomex sheets for the electrical insulation and is bound with Kevlar tapes for mechanical support against the repulsive electromagnetic force between two cables. The vacuum-insulated transfer line consists of four corrugated tubes assembled coaxially. Two-phase helium flows through the innermost channel, and returning helium gas flows through the outer annular channel as a thermal shield.

To guarantee stability, the bus-line was designed cryostable at the operating current of $30 \mathrm{kA}$. The bus-line was designed with a safety criterion specifying that the maximum temperature rise after a quench should be less than $77 \mathrm{~K}$ when the current decays with a time constant of $20 \mathrm{~s}$.

\section{SHORT SAMPLE TESTS OF A SINGLE STRAND}

We conducted short sample tests of one strand of the compacted nine-strand cable preceding the tests of the busline. Fig. 2 shows the measured and calculated minimum propagation currents of a strand with a cooling perimeter of $11.4 \mathrm{~mm}$ (37.5\% of the total perimeter of a strand). The calculations were done according to Maddock's equal area 
TABLE 1 SPECIFICATIONS FOR A SUPERCONDUCTING BUS-LINE

\begin{tabular}{ll}
\hline & Superconducting Bus-Line \\
\hline Operating current & $30 \mathrm{kA}$ \\
Breakdown voltage & $\mathrm{DC} 2 \mathrm{kV}$ (design) \\
(in helium gas at latm and $77 \mathrm{~K}$ ) & $>\mathrm{DC} 5 \mathrm{kV}$ (measured) \\
Length & $20 \mathrm{~m}$ \\
Maximum temperature risc after a & $<77 \mathrm{~K}$ with current decay time \\
quench calculated by hot-spot model & \multicolumn{1}{c}{ constant of 20 s } \\
\hline \multicolumn{2}{c}{ Aluminum Stabilized, Compacted Strand Cable } \\
\hline Critical current & $180 \mathrm{kA}$ at $1 \mathrm{~T}, 4.2 \mathrm{~K}$ \\
Size & $17.2 \mathrm{~mm} \times 35.4 \mathrm{~mm}$ \\
Operating current density & $53.0 \mathrm{~A} / \mathrm{mm}^{2}$ \\
Number of strands & 9 \\
Twist pitch of strands & $374 \mathrm{~mm}$ \\
Cooling perimeter & $35.4 \mathrm{~mm}$ \\
Minimum propagation current & $>30 \mathrm{kA}$ \\
\hline & Superconducting Strand \\
\hline Outer diameter & $8.95 \mathrm{~mm}$ \\
NbTi/Cu insert diameter & $2.91 \mathrm{~mm}$ \\
Al:Cu:NbTi ratio & $8.4: 0.5: 0.5$ \\
Al resistivity & $3 \times 10^{-11} \Omega \mathrm{m}$ at $1 \mathrm{~T}, 4.2 \mathrm{~K}$ \\
\hline
\end{tabular}

theorem [3] using the measured strand resistance and boiling heat transfer. (An aluminum resistivity derived from the measured strand resistance was $3 \times 10^{-11} \Omega \mathrm{m}$ which is the same as the design value.) Below $2 \mathrm{~T}$, the measured minimum propagation currents coincide with the calculated values. Above $2 \mathrm{~T}$, the minimum propagation current exceeds the critical current. When the current exceeds the critical current, the current transfers from the NbTi insert to the surrounding pure aluminum. However the cooling power exceeds the heat generation and the self-field at the $\mathrm{NbTi}$ insert decreases because of the current transfer to the surrounding aluminum. Therefore, a singular oscillation phenomenon was observed between the normal transition and the recovery to the superconducting state. The measured minimum propagation current was taken as the current when this oscillation stopped and jumped to the normal state.

Therefore the measured minimum propagation current above 2 $T$ was imprecise and differs from the calculation.

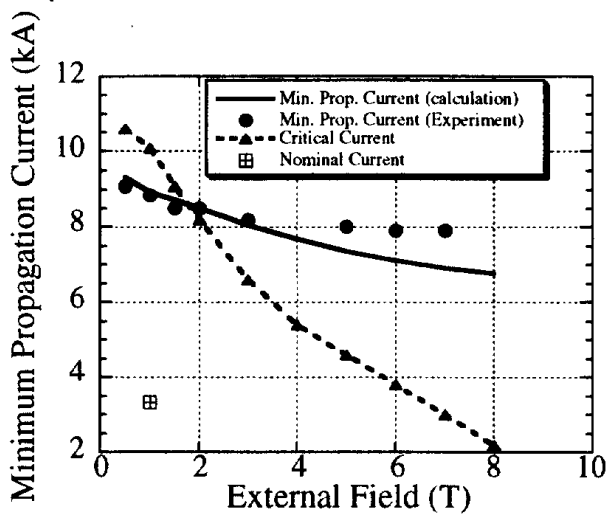

Fig. 2. Measured and calculated minimum propagation currents for a single strand.

\section{STABILITY TESTS OF THE SUPERCONDUCTING BUS-LIN}

\section{A. Experimental Set-up}

Two kinds of heaters ( $\mathrm{H} 1$ and $\mathrm{H} 2)$ were placed on the cable at the center of the bus-line. $\mathrm{Hl}$ is a resistive film heater attached to one strand of the cable. $\mathrm{H} 2$ is a stainless-steel foil heater which covers a flat surface of the cable.

An excitation test of the bus-line was carried out under short-circuit condition. The flow rate of the two-phase helium was kept constant at 11-12 g/s. We conducted successful overcurrent operation of $40 \mathrm{kA}$ without training.

\section{B. Stability Tests}

The stability margin and the minimum propagation current were measured by adding perturbation energies with heater $\mathrm{H} 2$ under the condition of the bus-line's self field. The input signal of the heater had a rectangular pulse with a duration of $1 \mathrm{~s}$. Keeping the bus-line transport current at a constant value, we increased the heater energy step by step. Fig. 3 shows the stability margin as a function of the perturbation energy required to initiate a quench. Below the current of 32.5 $\mathrm{kA}$, the initiated normal zone recovered even with the maximum heat input energy of $80 \mathrm{~J}$. Fig. 4 shows short voltage taps signals when the normal zone propagated at a transport current of $35 \mathrm{kA}$ and the heat input energy was $60 \mathrm{~J}$. The heater was attached inside the voltage taps V7V8. The short voltage taps have a sensitive length of about $100 \mathrm{~mm}$ and were attached at an interval of one strand twist pitch (374 $\mathrm{mm}$ ) of the superconducting cable. The voltage signals increased with two steps and then reached a plateau. The conductor resistance corresponding to this plateau was 5.9 $6.5 \times 10^{-8} \Omega / \mathrm{m}$ which nearly equals the design value. The first step voltages, which were about half of the plateau levels, indicate current sharing from quenched strands to other strands. When the normal zone recovered at a transport current of $35 \mathrm{kA}$ and the heat input energy was $50 \mathrm{~J}$, the voltage signals of V7V8 and V9V10 did not exceed the first step level of Fig. 4 and recovered to the superconducting state.

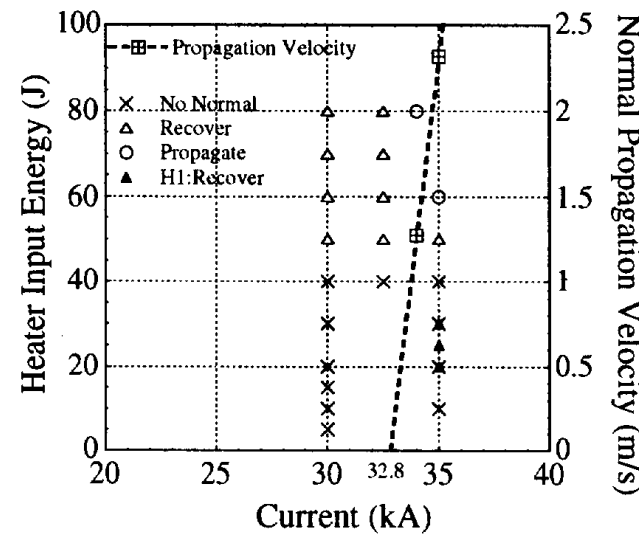

Fig. 3. Stability margin of the bus-line as a function of the perturbation energy required to initiate a quench. 


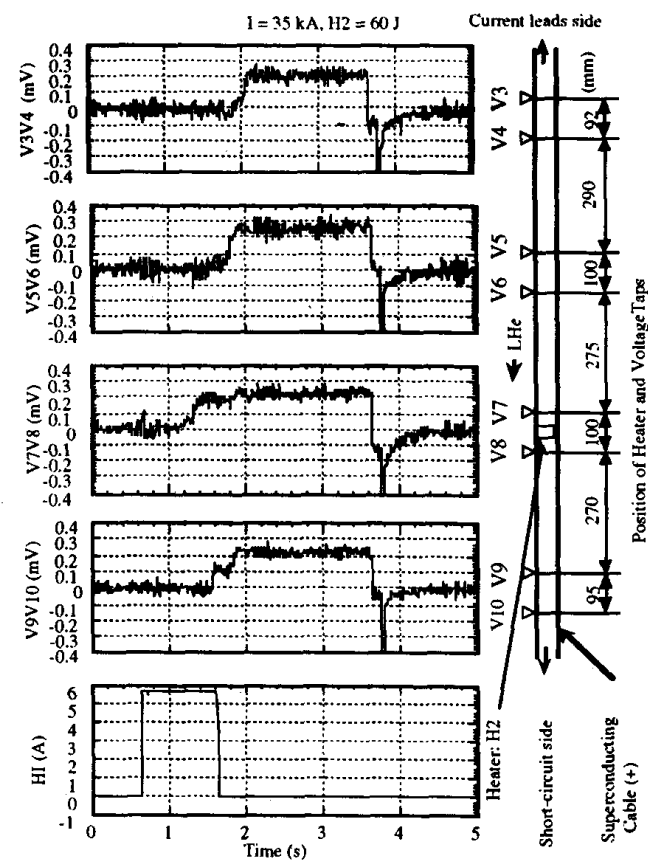

Fig. 4. Short voltage signals when the normal zone propagated at a transport current of $35 \mathrm{kA}$ and the heat input energy of $\mathrm{H} 2$ heater was $60 \mathrm{~J}$.

In Fig. 3, the stability margin measured with heater $\mathrm{Hl}$ (the heater attached to one strand of the nine-strand cable) is also shown. Keeping the operating current at $35 \mathrm{kA}$, we increased the heater $\mathrm{H} 1$ energy step by step in the same manner as that of the heater $\mathrm{H} 2$. Up to the heater limit of 30 $\mathrm{J}$, the initiated normal zone recovered to the superconducting state. It is considered that the current sharing from quenched strands to other strands can increase the stability of a multistrand superconducting cable.

Fig. 5 shows total voltage signals (for a sensitive length of $10 \mathrm{~m}$ ) of the normal propagation at the operating currents of $34 \mathrm{kA}$ and $35 \mathrm{kA}$. The voltage signals increased linearly

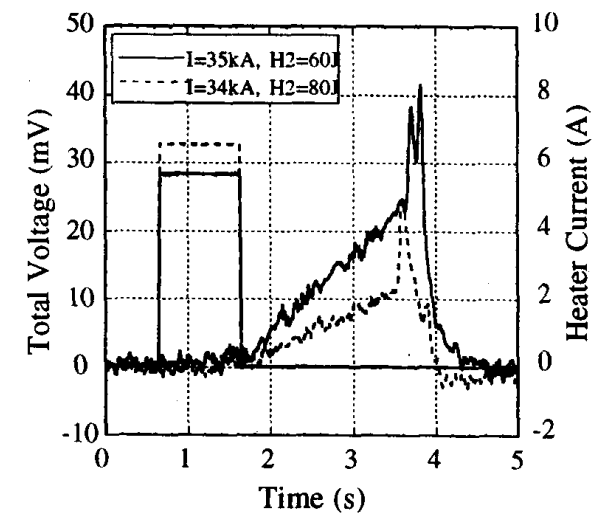

Fig. 5. Total voltage signal (for a sensitive length of $10 \mathrm{~m}$ ) of the normal propagation at the operating currents of $34 \mathrm{kA}$ and $35 \mathrm{kA}$.

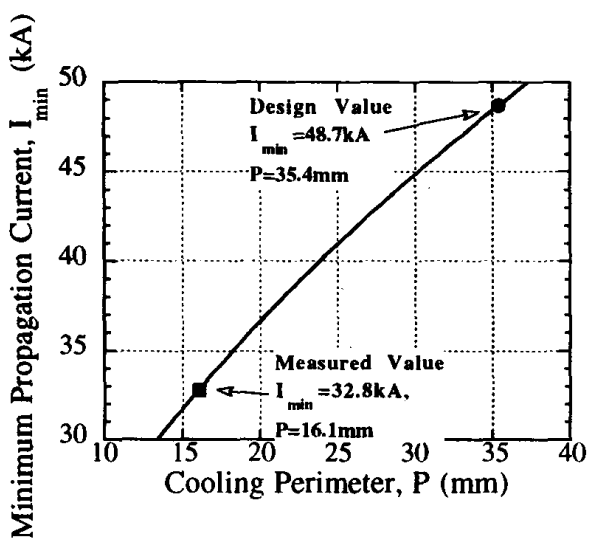

Fig. 6. Calculated minimum propagation current as a function of cooling perimeter for the superconducting bus-line.

after $2 \mathrm{~s}$, so that we could estimate the normal zone propagation velocities and plot them in Fig. 3. The normal propagation velocity increases linearly with the operating current. The minimum propagation current estimated from the cross point where the normal propagation velocity becomes zero is $32.8 \mathrm{kA}$.

\section{ESTIMATES OF STABILITY AND SAFETY}

\section{A. Estimate of Stability Including a Cooling Perimeter}

Fig. 6 shows the calculated minimum propagation current as a function of cooling perimeter for the superconducting bus-line. The design value of the cooling perimeter was set as $35.4 \mathrm{~mm}$ which corresponds to the flat surface of the superconducting cable that is not covered with an electrical insulator. The calculated minimum propagation current for this cooling perimeter $(35.4 \mathrm{~mm})$ is $48.7 \mathrm{kA}$. The cooling perimeter corresponding to the measured minimum propagation current $(32.8 \mathrm{kA})$ is $16.1 \mathrm{~mm}$, which is half of the design value. The decrease of the cooling perimeter is caused by the large heat input required to initiate a forced quench. The amount of liquid helium in the innermost channel is such that a heater input of $80 \mathrm{~J}$ fills a $76 \mathrm{~mm}$ length of the innermost channel with vaporized helium. Assuming no heat input, the minimum propagation current is $48.7 \mathrm{kA}$, or 1.62 times larger than the operating current of 30 $\mathrm{kA}$.

\section{B. Estimate of Safety with a Hot Spot Model}

Fig. 7 shows the calculated maximum temperature rises of the bus-line as a function of the current decay time constant. We calculated them with a hot spot model [5] using a measured resistance of the superconducting cable. At the design current decay time of $20 \mathrm{~s}$, the maximum temperature rise is $45.8 \mathrm{~K}$. The limit of a decay time, when the maximum temperature becomes $77 \mathrm{~K}$ (which was decided to prevent electric break down in helium gas), is $30 \mathrm{~s}$. The bus-line has a safety margin of $10 \mathrm{~s}$ in terms of decay time. 


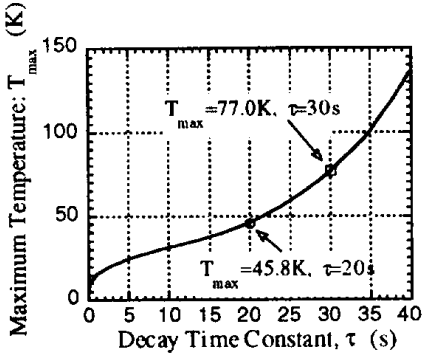

Fig. 7. Calculated maximum temperature rises of the bus-line as a function of the current decay time constant (the operating current density is $53 \mathrm{~A} / \mathrm{mm}^{2}$ ).

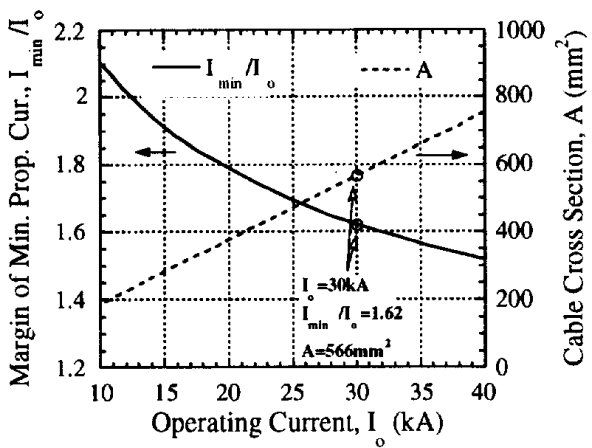

Fig. 8. Stability margin (the ratio of minimum propagation current to operating current) and cross section area as a function of operating current, keeping the operating current density constant at $53 \mathrm{~A}^{2} \mathrm{~mm}^{2}$.



Fig. 9. Maximum temperature rises with a hot spot model and cross section area as a function of the opcrating current, keeping stability margin constant at 1.62 .

\section{Design Criteria of Bus-Lines}

The evaluated stability and safety margin of this bus-line are sufficient and proper for the use of large-scale superconducting coils. We investigated the design criteria for a superconducting bus-line with operating current as a parameter based on this $30 \mathrm{kA}$ design. We assume that the basic superconducting cable design is not changed and is similar in shape. The composition and number of strands of a superconducting cable are the same and only the cross section area varies as a function of the operating current.

At first, we kept the safety margin the same as that of the $30 \mathrm{kA}$ design. Fig. 8 shows the stability margin (the ratio of a minimum propagation current to an operating current) and the cross section area as a function of operating current, keeping the operating current density constant at $53 \mathrm{~A} / \mathrm{mm}^{2}$. The safety margin is the same as shown in Fig. 7, if we keep a constant operating current density. The stability margin increases as the operating current decreases.

The calculated temperature rises with a hot spot model are overestimated since the cooling effect of liquid helium and the longitudinal heat conduction of the superconducting cable have been neglected. Then we keep the stability margin the same as that of the $30 \mathrm{kA}$ design. Fig. 9 shows the maximum temperature rises with a hot spot model and the cross section area as a function of the operating current, keeping the stability margin constant at 1.62 .

\section{CONCLUSION}

The stability and safety of the superconducting bus-line were confirmed experimentally, and their margins were evaluated. The measured minimum propagation currents of a single strand coincide with the calculated values using Maddock's equal area theorem. The measured minimum propagation current of the bus-line was $32.8 \mathrm{kA}$, which is decreased from the design value of $48.7 \mathrm{kA}$. This degradation of the stability can be explained as the decrease of the cooling perimeter from the design value of $35.4 \mathrm{~mm}$ to $16.1 \mathrm{~mm}$. The decrease of the cooling perimeter is caused by the large heat input required to initiate a forced quench. Assuming no heat input, the minimum propagation current is $48.7 \mathrm{kA}$, or 1.62 times larger than the operating current of $30 \mathrm{kA}$. At the design current decay time of $20 \mathrm{~s}$, the calculated maximum temperature rise of the bus-line is $45.8 \mathrm{~K}$. The evaluated stability and safety margin of this bus-line are sufficient and proper for the use of large-scale superconducting coils. Based on this $30 \mathrm{kA}$ bus-line design, the necessary cross section areas of the bus-line cable are calculated as a function of operating current, under the constant safety margin condition or the constant stability margin condition.

\section{ACKNOWLEDGMENT}

The authors are indebted to Prof. A. Iiyoshi, Director General of NIFS for his continuous encouragement. The authors are also grateful to Prof. R. Tobler for his helpful suggestions.

\section{REFERENCES}

[1] O. Motojima K. Akaishi, K. Fujii, S. Fujiwara, S. Imagawa et al, "Physics and engineering design on the Large Helical Device," Fusion Engineering and Design, Vol. 20, 1993, pp. 3-14.

[2] T. Mito, S. Yamada, K. Takahata, N. Yanagi, H. Chikaraishi, A. Nishimura, S. Tanahashi, O. Motojima, J. Yamamoto, $\Upsilon$. Uede H. Hive, K. Ueda, I. Itoh, M. Ikeda, and I. Inoue, "Development and Tests of a Flexible Superconducting Bus-Line for the Large Helical Device," IEEE Trans. Magn., Vol. 30, 1994, pp. 2090-2093.

[3] M. N. Wilson, Superconducting Magnets, Oxford University Press., 1983. 